\section{A comparison of \\ lymph node \\ measurement \\ techniques in canine \\ lymphoma and use of \\ computed \\ tomography \\ measurements to \\ assess clinical \\ response}

\section{Juliette Preston ${ }^{1}$, Ana Lara-Garcia ${ }^{1}$, Angela Taylor ${ }^{1}$, Randi Drees ${ }^{1}$, Owen Davies ${ }^{1}$, Ignacio Rodriguez ${ }^{1}$, Stephanie Byrne ${ }^{1}$, \\ Piotr Tytro', Isabelle Desmas², Sofia Carvalho², Chiara Leo ${ }^{3}$, Anneliese Stell ${ }^{1}$}

1 The Royal Veterinary College, London, UK

2 Davies Veterinary Specialists, Hertfordshire, UK

3 Instituto Veterinario di Novara, Novara, Italy

\section{OBJECTIVES}

To compare contemporaneous clinical caliper and CT scan measurements of lymph nodes in canine lymphoma patients retrospectively and to assess whether the plane of CT measurement affected response categorisation.

\section{METHODS}

Lymph nodes were measured on CT scans in the plane of acquisition (POA) and using 3-Dimensional Multi-Planar Reconstruction (3DMPR). Caliper and CT measurements were compared. For dogs with sequential scans, POA and 3DMPR measurements were performed and modified VCOG lymphoma-adapted RECIST criteria applied. Response categorisation was compared.

\section{RESULTS}

For long-axis measurements overall, median CT POA and caliper measurements were not significantly different but median CT 3DMPR was $6 \mathrm{~mm}$ greater than caliper measurement $(p<0.0001)$. Median short-axis caliper measurement was larger than CT POA and 3DMPR by $11 \mathrm{~mm}$ and $8 \mathrm{~mm}$ respectively $(p<0.0001$ for both). Bland Altman plots demonstrated better agreement between clinical measurements and 3DMPR (versus POA); closest agreement was between short-axis caliper and CT 3DMPR measurements.

There was no difference in response categorisation between long-axis POA and 3DMPR measurements overall $(p=0.411)$, although individual discordant results were observed.

Long-axis caliper and POA measurements were not consistently related, whereas 3DMPR measurements were greater than caliper measurement, perhaps because bilobed nodes were not detected clinically. Short-axis CT measurements were smaller than caliper measurements.

\section{STATEMENT}

Since caliper short-axis measurements agreed optimally with 3DMPR, this dimension might be preferred for caliper measurement. Overall, POA versus 3DMPR measurements did not affect response classification, thus easily attained POA measurements seem applicable; although individual discordant results occurred. A larger study is needed to standardise response assessment by $\mathrm{CT}$ in canine lymphoma. 